\title{
La renaissance du nu antique à Venise : Pietro Bembo et le Titien
}

Emilie Séris (Université Paris IV-Sorbonne)

The nude appears in Renaissance painting and literature on several occasions, in the wake the recovery of many ancient masterpieces both literary and visual, as well as the resulting humanist reappraisal of the body. Its natural splendour resurfaced with special acclaim in Venice, most particularly in the painted works of Titian. Pietro Bembo's Carmina also shed new light on the topic of nudity. Comparison of these poems and Titian's art unveils a series of shared commonplaces, namely, Cupid as the naked child, the female nude bathing (Diana, Galatea) or in the privacy of her room (Danae, Venus) and the male nude in the form of both a Christian martyr (Saint Stephen, Saint Sebastian) and a pagan divinity (Pan, Dionysos). At once in their written and painted manifestations, these nudes correspond closely to the aesthetic precepts explicated by two contemporary theoreticians of art and literature, Lodovico Dolce and Pietro Aretino. The essential precepts are : softness, delicacy and strength, terribilità and grace.

Le "Pastorum chorus" ("Le chœur des bergers") adressé au dieu Pan ouvre les Carmina de Pietro Bembo en chantant le retour du printemps. L'humaniste vénitien renouvelle ce topos affectionné des poètes de la Renaissance en peignant l'image d'un corps féminin qui se dénude: "iam collis uiret et sinu solutol/ florentes tibi porrigit genistas" 2 . Alors que chez la plupart de ses pairs, notamment florentins ${ }^{3}$, la terre se pare de ses bourgeons et de ses fleurs comme d'autant de gemmes et que la prairie émaillée lui est un habit brodé de toutes les couleurs, chez Bembo, la verte colline dénoue son manteau d'hiver et révèle ses appâts naturels.

Â la fin du Moyen Âge, la redécouverte de statues antiques intégralement nues ${ }^{4}$ ainsi que de textes anciens faisant l'éloge de la nudité a stimulé l'imaginaire des poètes et des artistes. Le développement de la médecine et de l'anatomie, l'intérêt nouveau pour l'hygiène corporelle ou la gymnastique ont amené à exalter la beauté du corps ${ }^{5}$. L'idéalisation de la femme, initiée par la littérature chevaleresque ${ }^{6}$, et la féminisation du public se passionnant pour les arts ont aussi concouru à l'avènement du nu féminin, quelque peu négligé dans l'Antiquité et à l'époque médiévale. Si la critique d'art s'est longtemps focalisée sur l'invention de la perspective et sur la théorie optique, de plus en plus d'études visent à revaloriser dans l'art italien de la Renaissance l'importance des sens autres que la vue et de la relation au

\footnotetext{
${ }^{1}$ La source de l'expression sine soluto est Columelle, Rei rustica liber X, 361 : sed resoluta sinus, resoluto masta capillo.

${ }^{2}$ Nous citons dans cet article l'édition du texte la plus récente : Pietro Bembo, Carmina, Turin, RES, 1990 (ici, I, 9-10, p. 5). Sur l'histoire du Carminum libellus, voir M. Pecoraro, Per la storia dei Carmi del Bembo- Una redazione non vulgata, Venezia-Roma, Istituto per la collaborazione culturale,1959, le compte-rendu de cet ouvrage par C. Dionisotti dans le Giornale Storico della Letteratura Italiana, 138 (1961), p. 573-592 ainsi que C. Dionisotti, "Per la storia del Carminum Libellus", Italia Medioevale e Umanistica, 8 (1965), p. 278-291 et Scritti sul Bembo, Turin, 2002, p. 101-113.

${ }^{3}$ Par exemple Cristoforo Landino, Xandra, 1, 3 (A. Perosa, Florence, 1939, p. 3-6) ; Politien, Rusticus, 172-282 (F. Bausi, Florence, 1995, p. 61-72) ; Marulle, Hymni naturales, 2, 7 (D. Coppini, p. 98-104) ; Tito Strozzi, Eroticon, 4, 8 :"Laus ueris ad Syluiam" (Venise, Alde Manuce, 1513).

${ }^{4} \mathrm{~K}$. Clark rapporte par exemple le vif émoi causé par l'exhumation d'un nu antique à Sienne au milieu du XIV ${ }^{\mathrm{e}}$ siècle dans la fameuse étude The Nude, Princeton University Press, 1956, p. 88-89 (trad. M. Laroche, Hachette, 1998, tome I, p. 154-155).

${ }_{5}^{5}$ Cf. N. Laneyrie-Dagen, L'invention du corps. La représentation du corps du Moyen-Age à la fin du XIXe siècle, Paris, Flammarion, Tout l'Art, 2006.

${ }^{6}$ L'importance inattendue de la nudité dans la littérature médiévale a été révélée dans Le nu et le vêtu au MoyenÂge (XVIIe-XIIIe siècles), actes du $25^{\mathrm{e}}$ colloque du Centre Universitaire d'Etudes et de Recherches Médiévales d'Aix, Aix-en-Provence, 2001.
} 
corps $^{7}$. Or, c'est assurément à Venise, avec l'art de la couleur de Giorgione, du Titien et du Tintoret, que la sensualité du corps est célébrée avec le plus de bonheur'.

C'est à Venise aussi que l'Arétin et Lodovico Dolce, deux critiques d'art proches du Titien ${ }^{9}$, ont ébauché les premiers préceptes de ce qui deviendrait au siècle suivant une théorie du nu ${ }^{10}$. Louant les nus de Raphaël, Dolce propose dans le Dialogo della pittura intitolato l'Aretino (1557) une première classification du genre en trois types à la fois fondés sur des distinctions physiologiques et légitimés par la convenance : "Fece i putti putti, cioè morbidetti e teneri : gli huomini robusti, e le donne con quella delicatezza, che convien loro"11. Les trois sortes de nus enfantin, masculin et féminin sont respectivement identifiés par les concepts esthétiques de douceur, de robustesse et de délicatesse. Plus loin, opposant les nus de Raphaël à ceux de Michel-Ange, il propose une seconde classification en fonction des affects suscités chez le spectateur: "E da avertir, che Michel'Agnolo ha preso del nudo la forma piu terribile e ricercata, e Rafaello la piu piacevole e gratiosa"12. On sait, grâce à la récente étude de Thomas Puttfarken sur peinture et tragédie chez Le Titien ${ }^{13}$, l'influence dans le milieu vénitien de la Poétique d'Aristote, publiée en traduction latine par Giorgio Valla à Venise en 1498, et plus particulièrement de sa théorie de la catharsis. Le nu est donc aussi apprécié en fonction de sa puissance émotive et de sa capacité à éveiller la terreur ou au contraire le plaisir.

Chaque fois qu'il aborde la question du nu, Lodovico Dolce invoque expressément le paragone, comme lorsqu'il vante cette qualité que, seul selon lui avant Raphaël et Titien, le peintre Apelle avait su donner à ses figures: "La venustà, che è quel non so che, che tanto suole aggradire, cosi ne' Pittori, come ne'Poeti, in guisa, che empie l'animo altrui d'infinito diletto, non sapendo da qual parte esca quello, che a noi tanto piace" ${ }^{14}$. Pietro Bembo et Tiziano Vecellio ont cultivé une longue amitié : c'est l'humaniste, alors qu'il venait d'être nommé en 1513 secrétaire pontifical, qui lança la carrière de son compatriote en lui remettant en mains propres une invitation de Léon $\mathrm{X}$ qui faisait de lui le peintre officiel de la curie romaine $^{15}$. Si le Titien choisit de rester à Venise, il n'en témoigna pas moins son estime et sa reconnaissance à l'écrivain en tirant à plusieurs occasions son portrait ${ }^{16}$. La précédente formule de Dolce a été pour moi une incitation et une autorisation à chercher précisément, dans les correspondances entre les poèmes de Bembo et les toiles du Titien, ce "non so che"

\footnotetext{
${ }^{7}$ Cf. D. Summers, The Judgement of Sense : Renaissance Naturalism and the Rise of Aesthetics, Cambridge, 1987 ; S. Ferino-Pagden, I cinque sensi nell'arte. Immagini del Sentire, cat. de l'exposition du Centro culturale "Citta di Cremona" (21 septembre 1996-12 janvier 1997), Venezia, Leonardo Arte, 1996 et, plus récemment, François Quiviger, The Sensory World of Italian Renaissance Art, London, 2010.

${ }^{8}$ Cf. S. Ferino-Pagden, L'ultimo Tiziano e la sensualità della pittura, Venezia, Marsilio, 2008 et A. Galansino, "La femme offerte. Peintures érotiques à Venise", dans Titien, Tintoret, Véronèse. Rivalités à Venise, catalogue de l'exposition du Musée du Louvre (17 septembre 2009-4 janvier 2010) Paris, Hazan, 2009, p. 296-317.

9 Titien s'est lié avec le poète toscan Pietro Aretino dès l'arrivée de celui-ci à Venise, en mars 1537 . Il le reçut régulièrement dans sa maison de Biri Grande, avec l'architecte également toscan Sansovino, et peignit son portrait en 1445 (cf. F. Pedrocco, Tiziano, Milan, 2000, trad. française p. 47-55 et B. Cole, Titian and Venetian's Painting, 1450-1590, Oxford, 1999, p .136-139). Comme l'Arétin, le polygraphe vénitien Lodovico Dolce connaissait personnellement le peintre (B. Cole, p. 63) et fut son virulent défenseur. Le Dialogue de la peinture apparaît dans une certaine mesure comme une réponse à Giorgio Vasari, qui avait minoré dans ses Vies la place du Titien parmi les peintres vénitiens (P. Meilman, Titian and the Altarpiece in Renaissance Venice, Cambridge, 2000, p. 133-137). Les écrits de Dolce et de l'Arétin demeurent les témoignages historiques les plus précieux que nous ayons sur la vie et l'œuvre du peintre.

${ }^{10}$ Sur les débuts de la théorie de l'art à Venise, voir M. Hochmann, Peintres et commanditaires à Venise (15401628), Rome, EFR, 1992, "Peintres et lettrés", en particulier p. 123-127 et 136-143.

${ }^{11}$ Lodovico Dolce, Dialogo della pittura intitolato l'Aretino, éd. Mark W. Roskill, dans Dolce's "Aretino" and Venetian art Theory of the Cinquecento, New York University Press, 1968, p. 160, trad. L. Fallay d'Este, Paris, 1996, p. 86 : "il fit des putti pareils à des putti, c'est-à-dire doux et tendres, et attribua aux hommes la robustesse qui leur convient, aux femmes une juste délicatesse".

${ }_{12}$ Ibidem, éd. M.W. Roskill, p. 172 et trad. L. Fallay d'Este, p. 90 : "Il faut préciser que Michel-Ange a pris du nu la forme la plus recherchée, la plus empreinte de terribilità, et que Raphaël a choisi la plus agréable et la plus gracieuse."

${ }_{13}$ Cf. T. Puttfarken, Titian \& tragic painting. Aristotle's Poetic and the Rise of the Modern Artist, New HavenLondon, Yale University Press, 2005.

${ }^{14}$ Lodovico Dolce, Dialogo della pittura intitolato l'Aretino, éd. M.W. Roskill, p. 174 et trad. L. Fallay d'Este, p. 92 : "La vénusté, qui consiste, aussi bien chez les peintres que chez les poètes, en ce je ne sais quoi si agréable qui remplit l'esprit de plaisir, bien qu'on en ignore l'origine".

${ }^{15}$ Cf. J. Pope-Henessy, Titien, Paris , Gallimard, 2004, p. 87.

${ }^{16}$ Le célèbre portrait conservé à la National Gallery de Washington a été réalisé peu après la nomination de Pietro Bembo comme cardinal en 1539. Le dernier portrait daterait de son séjour à Rome en 1445-1446, c'est-àdire des dernières années de sa vie : c'est aussi l'époque ou le Titien a tiré de le portrait de l'Arétin.
} 
qui fait la vénusté des nus vénitiens ${ }^{17}$. Me fiant au jugement de ce grand esthète, j'étudierai quelques exemples de nus enfantins, féminins et masculins qui me paraissent illustrer dans les Carmina et dans la peinture du Titien les notions de "morbidezza", de "robustezza" et de "delicatezza", de "terribilità" et de "grazia".

\section{I -L'Amour nu}

Dans une élégie intitulée "Amica ad Gallum"18, Pietro Bembo reprend le thème ancien du "nudus puer". On sait que l'identification de l'Amour à un enfant nu trouve une légitimation dans la tradition philosophique platonicienne. Dans le Banquet de Platon, l'Amour, qui est désir de la beauté, est nu parce qu'il est fils de Pénia, l'indigence. Marsile Ficin donne dans le De Amore (1469) une double signification physique et morale à sa nudité. L'Amour est à la fois "doux, délicat et tendre" ("mollem, delicatum et tenerum"), ce qui est "l'indice d'un heureux dosage entre les quatre humeurs"19. De plus, "l'Amour est libre et naît spontanément dans une volonté libre" car celui qui aime ne désire rien d'autre de l'aimé que son amour $^{20}$. Pietro Bembo, pour avoir transmis ces doctrines dans les Azolains, connaissait parfaitement ces interprétations associant la nudité d'Amour au dénuement du désir, à l'heureuse complexion du corps ou à la liberté morale de l'amant. Plusieurs études ont montré aussi l'influence du néo-platonisme sur l'invention des toiles du Titien et en ont imputé la principale responsabilité à Bembo lui-même ${ }^{21}$.

Toutefois, la source du passage se trouve chez Ovide. Dans l'élégie I, 10 des Amores, l'amant fustige une de ses maîtresses parce qu'elle lui a demandé de l'argent en échange de ses privautés. "L'Amour est enfant et nu" ("Et puer est et nudus Amor") $)^{22}$, lui répond-il, car il est sans ruse et sans avarice. Chez les poètes élégiaques latins, la nudité de l'amour est la preuve de sa sincérité et de son désintéressement ${ }^{23}$. Jeunesse et absence de vêtement révèlent l'incapacité du véritable amour à dissimuler ou à tromper. Cupido n'est pas cupiditas et l'élégie d'Ovide s'achève sur une condamnation vigoureuse de l'amour vénal. Au Moyen-Âge, l'amour courtois a encore approfondi la différence entre l'amour vrai et l'avarice, et renforcé le blâme pesant sur la prostitution ${ }^{24}$ Grandi dans une cité célèbre pour ses courtisanes, Pietro Bembo s'amuse à renverser les points de vue en intervertissant la situation d'énonciation ; c'est une jeune femme qui adresse à son amant des récriminations, accusant les hommes de sacrifier leurs maîtresses à leur ambition :

Adde quod est Amor ipse puer, nec conuenit illo

${ }^{17}$ Les travaux de R. W. Lee sur le paragone entre poésie et peinture (Ut Pictura Poesis. The Humanistic Theory of Painting, Norton and Company, 1967) et ceux de M. Baxandall sur l'adaptation de la rhétorique en peinture (Giotto and the Orators. Humanist Observers of painting in Italy and the Discovery of Pictorial Composition, London, Oxford University Press, 1971 et Painting and Experience in Fifteenth Century Italy, London Oxford Universtity Press, 1972) ont bien montré les liens étroits tissés par les humanistes entre littérature et art.

18 Sur les élégies de Pietro Bembo, voir l'article de John N. Grant dans un volume précédent de la présente revue: "Propertius, Ovid an Two Latin Poems of Pietro Bembo", International Journal of the Classical Tradition, spring 1995, vol. 1, Issue 4, p. 48-62. Comme le souligne l'auteur, peu nombreuses ont été en général les études poétiques sur les Carmina de Pietro Bembo. On retiendra l'article de I. Reineke sur le Benacus ("Der 'Benacus' des Pietro Bembo", Res publica litterarum, 12 (1979), p. 177-183) et celui de J. Ijsewijn sur le Pro Corytio, ("Poetry in a Roman Garden : The Coryciana", dans Latin Poetry and the Classical tradition: Essays in medieval and Renaissance Poetry, dir. P. Godman et O. Murray, Oxford, 1990, p. 211-231).

${ }^{19}$ Marsile Ficin, De Amore, V, 7, éd. et trad. P. Laurens, Paris, Les Belles Lettres, 2002, p. 108-111.

${ }^{20}$ Ibidem, V, 8, p. 114-115.

${ }^{21}$ Cf. E. Panofsky, Problems in Titian mostly iconographic, London-New York, Phaidon, 1969, p. 120 (Le Titien. Questions d'iconographie, Paris, Hazan, 2004, p. 164); A. Gentili, Da Tiziano a Tiziano. Mito e allegoria nella cultura veneziana del Cinquecento, Roma, Feltrinelli, 1980, p. 81-87 et U. Roman d'Elia, The poetics of Titian's religious paintings, Cambridge, 2005, "Titian and Writers", p. 5-8.

22 Ovide, Amores, I, 10, 15.

${ }^{23}$ Comme l'a montré S. L. James, l"opposition entre amour et argent est l'un des lieux fondateurs de l'élégie et se rattache plus généralement à la recusatio de l'épopée, c'est-à-dire au refus d'une poésie officielle et rémunérée (Learned Girls and Male Persuasion. Gender and Reading in Roman Love Elegy, Berkeley-Los AngelesLondon, University of California Press, 2003 et en particulier "Voluntary poverty and the elegiac recusatio", p. 72-98). Le thème se retrouve notamment chez Ovide $(1,3 ; 1,15$ et 2,1$)$, Properce $(1,7$ et 1,9$)$ et Tibulle $(1$, $4 ; 1,8$ et Corpus tibull. 1, 1, 7).

${ }_{24}$ Cf. Andreas Capellanus, De amore, I, 2 : : cunctis constat [auaritiam] in eodem cum amore non posse domicilio permanere (trad., G. Buridan, Paris, Klincksieck, 1974: "il est bien clair pour tous les hommes que l'avarice ne peut cohabiter avec l'amour") et I, 4: Effectus amoris hic est, quia uerus amator nulla posset auaritia offuscari (trad. G. Buridan : "L'amour fait que le véritable amant ne peut être avili par l'avarice"). 
Versutum pectus militis esse duce.

Vtque deus nullo uelat sibi corpus amictu,

Sic nudis animis uult sua bella geri :

Quemque uidet non insidiis, non artibus uti,

Hic bonus est, inquit, miles, eritque mihi.

De plus, Amour lui-même est un enfant, et sous ses ordres avoir le cœur rusé ne sied pas au soldat.

De même que le Dieu ne voile son corps d'aucun habit,

De même, il veut que l'on mène ses combats l'âme nue.

Et celui qu'il ne voit pas user d'embûches ni d'artifices,

Il est bon soldat, dit-il, et le sera pour moi. ${ }^{25}$

Pratiquant à son tour l'exégèse allégorique, le poète néo-latin justifie le jeune âge et la nudité de Cupidon : il est sans détour et ne cherche pas à esquiver les coups. Il lutte à mains nues et en combat loyal. Dans la bouche de la jeune femme, Amour ne s'oppose plus par sa nudité à la cupidité et à la prostitution, réservées au beau sexe, mais au désir de puissance et de gloire des hommes. Sous prétexte de servir les leurs, les princes, ou la patrie, ils abandonnent les jeunes filles qu'ils ont séduites et causent leur perte.

Titien, plus qu'aucun peintre auparavant, s'est attaché à imiter le corps enfantin avec toutes les particularités de proportions, d'attitudes, la rondeur des formes et la finesse de la chair caractéristiques de cet âge de la vie ${ }^{26}$. Il a peint, parmi tant d'autres, un Amour nu dans la première Danae réalisée à Rome pour Ottavio Farnese dans les années 1444-1446 et conservée à Naples (Fig. 1). Les critiques s'accordent généralement sur la fonction du petit dieu dans l'invention du tableau : il persuade le spectateur que Danae s'est donnée à Jupiter, métamorphosé en pluie d'or, par amour et non par cupidité. En effet, Cupidon se détourne de l'averse de pièces qui tombe sur la couche de la belle. Son visage et le geste du bras par lequel il cherche à se protéger disent assez son aversion pour l'argent. Amour nu sauve la morale dans un tableau dont le pouvoir érotique avait frappé Michel-Ange lui-même. On sait que dans les versions suivantes, notamment celle peinte pour le camerino de Philippe II d'Espagne en 1552, la figure d'Amour nu fut remplacée par une servante âgée, noire et totalement vêtue, qui se précipite pour récupérer dans son tablier les pièces d'or. La signification du tableau s'en trouve profondément altérée.

\section{-la morbidezza}

Pietro Bembo a peint aussi la troupe des Amours à la fin du "Pastorum chorus". La fête du printemps est, comme de juste, présidée par Vénus, accompagnée de son cortège de Grâces et d'allégories (la Jeunesse, le Badinage et la Volupté). L'image attendrissante de la déesse embrassant les Amours nus donne une bonne idée, me semble-t-il, de ce que peut signifier la "morbidezza" en poésie.

Gregi hac poscimus : at gregis magistros

Prosens perpetua leues iunenta,

Quam lusus comitetur et uoluptas

Et nudos Venus osculans amores.

Nous le demandons pour le troupeau : mais, jeunesse éternelle, favorise et soulage aussi les maîtres du troupeau,

Toi qu'accompagnent le badinage et la volupté,

\footnotetext{
${ }^{25}$ Pietro Bembo, Carmina, XIII : "Amica ad Gallum", $83-88$ (Turin, 1990, p. 27). Je donne mes traductions des poèmes de Pietro Bembo.

${ }^{26}$ Anthony Colantuono a longuement analysé l'invention, chez Titien, d'une nouvelle figure de putto caractérisé par sa tenerezza et qui devait influencer par la suite des artistes comme Duquesnoy et Poussin (The Tender Infant: 'Invenzione' and 'Figura' in the Art of Poussin, Ph. D. dissertation, The Johns Hopkins University, 1986, p. 222-275 ; "Titian's Tender Infants. On the Imitation of Venetian Painting in Baroque Rome", I Tatti Studies, 3 (1989), p. 207-234. Au XVII siècle, le critique d'art G.P. Bellori a défini le putto moderno inventé par Titien par opposition au putto antico des Anciens et de Raphaël comme un enfant plus jeune (dans sa première année), à l'anatomie immature et en réalité impropre à effectuer les actions dans lesquelles il était représenté par le peintre. L'ensemble de ces qualités avait pour effet de susciter la tendresse et l'amour du spectateur. Ajoutons que peindre la prime enfance est un défi en raison de l'incapacité des très jeunes modèles à tenir une pose : ce n'est sûrement pas un hasard si c'est souvent dans le sommeil que Titien a saisi ses putti, comme par exemple dans le tableau intitulé Les trois âges de la vie.
} 
Le poète produit une impression de douceur grâce à une allitération en consonnes liquides (l, $\mathrm{m}$ et $\mathrm{n}$ ) et la répétition de l'harmonie vocalique (é-u-o) : "Et nudos Venus osculans amores". En effet, les poètes de la Renaissance, nourris de rhétorique cicéronienne, avaient appris la doctrine de la suauitas, qui s'obtient par la modulation des accents de la voix, l'arrangement judicieux des mots et leur liaison grâce au rythme ${ }^{28}$. Les humanistes connaissaient aussi les préceptes de Denys d'Halicarnasse ${ }^{29}$ ou d'Hermogène de Tarse ${ }^{30}$ sur l'euphonie et l'harmonie. Ainsi, Coluccio Salutati voyait dans le rythme et la mélodie la source de la douceur que produisent les vers à l'oreille ${ }^{31}$. Giovanni Pontano fait de l'allitération un procédé essentiel de uoluptas en poésie ${ }^{32}$. Plus largement, l'esthétique horatienne de la "suauitas" portait à la recherche d'une expression de la tendresse ${ }^{33}$. En peinture, comme le dit expressément Lodovico Dolce, la douceur ou "morbidezza", en particulier dans la représentation du nu, dépend de l'art de disposer les lumières et les ombres pour produire l'impression de relief :

Questi lumi et ombre posti con giudicio et arte fanno tondeggiar le figure : e danno loro il rilevo, che si ricerca : delqual rilevo le figure, che sono prive, paiono, come ben diceste, dipinte, percioche resta la superficie piana. Chi adunque ha questa parte, ne ha una delle piu importanti. Cosi la principal difficultà del colorito è posta nella imitation delle carni, e consiste nella varietà delle tinte, e nella morbidezza.

Placées avec jugement et art, les lumières et les ombres font ressortir l'arrondi des figures et leur donne le relief nécessaire ; et comme vous l'avez bien dit, les figures sans relief semblent peintes, puisque la superficie reste plane. Ainsi, celui qui possède cette qualité, possède l'une des qualités les plus importantes. Et la difficulté majeure des couleurs réside dans l'imitation des chairs, et consiste dans la variété des teintes et de la douceur. ${ }^{34}$

Parmi les bacchanales peintes par le Titien pour le studiolo d'Alfonse d'Este entre 1518 et 1523, l'une, connue sous le nom de d'Offrande à Vénus (Fig. 2), avait pour sujet la "fête des Amours" telle qu'elle est décrite par Philostrate dans les Imagines (I, 6, "Amores") : le tableau antique était une allégorie de l'amour réciproque ${ }^{35}$. Le peintre a suivi à la lettre l'écrivain selon lequel les Amours s'étaient dépouillés de leurs vêtements et de leurs carquois pour s'adonner à la récolte des pommes : en effet, des carquois apparaissent suspendus aux branches des arbres et des habits froissés jonchent le sol au premier plan. La foule des putti jouant est une

\footnotetext{
${ }^{27}$ Pietro Bembo, Carmina, I : "Pastorum chorus", 79-82 (Turin, 1990, p. 8).

${ }^{28}$ Cf. Cicéron, Orator, XVII-XVIII, 57-58 ; XLIV, 149-150 et LIV, 182.

${ }^{29}$ Cf. Denys d'Halicarnasse, De compositione uerborum, VI, 11 notamment. L'enseignement du De compositione uerborum a été transmis par Georges de Trébizonde (Rhetorices libri V, Venise, 1472). Le texte de Denys a ensuite été édité à trois reprises au début du Cinquecento (une édition s.d.l.n.., une à Paris, Estienne, 1547 et une à Strasbourg, J. Sturm, 1550). Sur l'influence de Denys d'Halicarnasse à la Renaissance, voir P. Laurens, "La performance stylistique dans le chapitre De numeris : J.C. Scaliger lecteur de Denys d'Halicarnasse" dans C. Balavoine et P. Laurens éd., La statue et l'empreinte. La poétique de Scaliger, Paris, Vrin, 1986, p. 131-150.

${ }^{30}$ Cf. Hermogène, De ideis, en particulier p. 307 (trad. M. Patillon, Hermogène de Tarse. Sur les Idées, Paris, L'âge d'homme, 1997, p. 408). Le texte d'Hermogène a été transmis au Quattrocento par plusieurs manuscrits conservés à Rome ou à Fiesole, par des incunables à Venise (1472) ou Milan (1493) et au début du Cinquecento par des éditions (éditio princeps par Alde Manuce à Venise en 1508 et par Georges de Trébizonde à Paris en 1520). Sur la transmission de la rhétorique d'Hermogène à la Renaissance, voir l'introduction de P. Laurens à l'édition citée.

31 Cf. Coluccio Salutati, De Herculis laboribus, I, 11.

${ }^{32}$ Cf. Giovanni Pontano, Actius, p. 120 r-121 v. Sur la notion de douceur liée au travail du rythme et de l'euphonie chez ces deux derniers auteurs, voir encore P. Laurens, "Trois lectures du vers virgilien (Coluccio Salutati, Giovanni Pontano, Jules-César Scaliger)", La dernière muse latine. Douze lectures poétiques, de Claudien à la génération baroque, Paris, Les Belles Lettres, 2008, p. 109-138.

${ }^{33} \mathrm{Cf}$. Horace, Art poétique, 333-346. Sur l'influence de la suauitas, quatrième vertu du poète chez Horace, à la Renaissance, voir P. Galand Hallyn et F. Hallyn, Poétiques de la Renaissance. Le modèle italien, le monde franco-bourguignon et leur héritage en France au XVI siècle, Genève, Droz, 2001, p. 507.

${ }^{34}$ Lodovico Dolce, Dialogo della pittura, éd. M. W. Roskill, p. 154 et trad. L. Fallay d'Este, p. 82.

35 A. Colantuono a proposé récemment une interprétation astrologique et cosmologique des Bacchanales de Ferrare fondée sur les Saturnales de Macrobe et sur leur relecture par Mario Equicola dans son Libro De Natura de Amore (Titian, Colonna and the Renaissance Science of Procreation. Equicola's Seasons of Desire, SurreyBurlington, Ashgate, 2010, p. 131-149). L'offrande à Vénus représenterait la saison de l'automne et les jeux des putti se lançant des pommes, l'égalité de la libido masculine et de la libido féminine à l'approche du solstice d'hiver. Cette analyse très cohérente n'élucide pas cependant la raison de la nudité affichée des Amours.
} 
démonstration magistrale de la technique du clair-obscur : le peintre parvient à rendre par le fondu des teintes le relief des plis de chair, potelés et tendres, des Amours ${ }^{36}$.

\section{II -La Néréide}

On trouve dans les Carmina de Bembo deux nus féminins très différents et bien caractérisés. Le premier est un sujet mythologique et représente une femme au bain (Carmina, VII : "Galatea", 53-66). Le second est un sujet élégiaque et représente une femme couchée dans l'intimité de sa chambre (Carmina, XV : "De amica a uiro seruata diligentissime", 1120). Ces deux séquences littéraires correspondent étonnamment à deux situations favorites du nu féminin chez Titien : la scène de bain (Vénus Anadyomène, Diane et Actéon, Diane et Callisto, L'enlèvement d'Europe, Persée et Andromède...) et la femme alitée (Vénus d'Urbin, Danae, Vénus et la musique, Tarquin et Lucrèce...).

On trouve une ravissante peinture de baigneuse dans la septième pièce des Carmina de Pietro Bembo, un poème qui appartient au cycle consacré à Faunus. Il s'agit d'une description de la néréide Galatée ${ }^{37}$. Bembo reprend le thème traité par Théocrite dans la bucolique XI, par Ovide dans les Métamorphoses (XIII, 789-808) et plus récemment par Ange Politien dans l'ode "In puellam suam" (Odae, VIII) et dans ses Stances (I, 115-118) ${ }^{38}$. Le poète vénitien introduit cependant une variante importante dans la fable puisque l'amoureux qu'elle éconduit n'est plus le cyclope Polyphème mais le dieu Pan lui-même ${ }^{39}$. L'attirant malicieusement dans les flots, la néréide cède finalement aux prières des serviteurs du Faune: elle le sauve in extremis de la noyade et le ramène inanimé sur le rivage :

Omnia qua obliquo, lente nans, respicit ore, Vnaque tot gaudet fallere Nympha Deos.

Vtque satis lusit : "Certe sat lusimus, inquit, Sucessuque mei non caruere doli".

Atque ita iactatis relegit freta uersa lacertis, Pronaque non longum uincere tendit iter.

Summa secant pulsa fluctu saliente papilla, Spumea sub niueo murmurat unda pede.

Elle, nageant lentement, le visage incliné en arrière, voit tout Et se réjouit d'abuser à elle seule, nymphe, autant de dieux.

Quand elle s'est suffisamment amusée : "Le jeu a assez duré". Dit-elle, "et ma ruse a eu un franc succès".

Alors elle se retourne et franchit les flots en battant des bras Et, plongeant, s'efforce de vaincre la brève distance.

Ses seins frappés par les flots coupent la surface de l'eau, L'onde écumeuse murmure sous son pied de neige. ${ }^{40}$

\footnotetext{
${ }^{36}$ D'après A. Colantuono, c'est précisément ce tableau qui aurait été à l'origine du type du putto moderno théorisé par G.P. Bellori et diffusé par la poésie de G. Marino, figure dont la fortune allait être immense à l'époque baroque ("Titian's Tender Infants...", p. 208 sqq et 223 sqq). Pourtant, du vivant même du Titien, bien avant que G.P. Bellori ne parle de la tenerezza de ces putti, Dolce avait défini la morbidezza du nu enfantin et Pietro Bembo en avait cherché un équivalent poétique grâce à la musique de ses vers.

${ }^{37}$ R.V. Young a fait une étude comparative de diverses réécritures du mythe de Polyphème et Galatée parmi lesquelles celle de Pietro Bembo: "Versions of Galatea: Renaissance and Baroque Imitation, Renaissance Papers, 1984, p. 57-67.

${ }_{38}$ On peut lire l'ode "In Puellam suam" de politien dans I. del Lungo, Poesie volgari inedite et poesie latine e greche edite e inedite, Florence, Barberà, 1867, p. 268-271 et en traduction française dans P. Laurens, Anthologie de la poésie lyrique latine de la Renaissance, Paris, Gallimard, 2004, p. 78-86. Le passage des stances de Politien consacré à Galatée a été édité par F. Bausi et traduit par E. Séris dans Ange Politien, Stances/Stanze et Fable d'Orphéel Fabula di Orfeo, Paris, Les Belles Lettres, 2006, p. 40-41. Sur la figure de Galatée dans la poésie de Politien, voir E. Séris, Les étoiles de Némésis : rhétorique de la mémoire dans la poésie d'Ange Politien (1454-1494), Genève, Droz, 2000, p. 381-414 et sur l'ode In Puellam en particulier, voir les articles de J.L. Charlet, "L'ode 8 de Politien : un hymne à la beauté ?", dans G. Tarugi, Homo sapiens, homo humanus, Florence, Olschki, 1990, vol. II, p. 41-56 et C. Fantazzi, "The Style of Quattrocento latin love Poetry", International Journal of the Classical Tradition, 1996, vol. 3, Issue 2, p. 127-147.

${ }^{39}$ Sur le personnage du Faune dans les Carmina de Pietro Bembo, voir E. Travi, "Notarella al 'Faunus' di Pietro Bembo", Lettere italiane, 33 (1981), p. 385-38 ; J.S. Salemi, "Priapus by Pietro Bembo", Allegorica 5 (1980), p. 81-94 et "The Faunus Poems of Pietro Bembo", Allegorica 7 (1982), p. 31-57. Pour une étude générale sur le mythe de Pan à la Renaissance, voir F. Lavocat, La Syrinx au bûcher, Pan et les satyres à la Renaissance et à l'âge baroque, Genève, Droz, 2005.

${ }_{40}$ Pietro Bembo, Carmina, VII : "Galatea", 53-60 (Turin, 1990, p. 15).
} 
Les modèles antiques et humanistes de Bembo prenaient déjà la chanson de Polyphème comme une occasion de dépeindre la beauté du corps de Galatée par une série de comparaisons animales, végétales ou minérales. Depuis Théocrite, un jeu étymologique sur le nom Galatée justifiait en particulier la comparaison de son teint au lait caillé (gala, galatos). La néréide avait à ce titre inspiré aussi les peintres et en 1511 Raphaël peignit une Galatée dans le palais du banquier Agostino Chigi devenu aujourd'hui la villa Farnésine. Bembo, qui a par ailleurs imité la chanson de Polyphème dans un autre poème (III : "Faunus ad Nymphas") ${ }^{41}$, esquisse de la néréide une figure en mouvement, saisissant avec précision ses gestes et dévoilant les parties de son corps qui surgissent des ondes quand elle nage : le visage tourné en arrière, les bras s'agitant, les seins qui fendent la surface de l'eau, le pied de neige qui soulève l'écume dans son sillage.

\section{-Robustesse et délicatesse}

Les écrits de l'Arétin et de Lodovico Dolce nous apprennent que deux critères essentiels pour apprécier les nus dans l'esthétique vénitienne de la première moitié du Cinquecento sont la robustesse et la délicatesse. Dolce, immédiatement après avoir distingué le nu du vêtu, précise qu'il existe deux manières de peindre le nu, musclé ou délicat :

Ora abbiamo a considerar l'huomo in due modi, cioè nudo e vestito. Se lo formiamo nudo, lo possiamo far di due maniere : cioè o pieno di muscoli, o delicato : laqual delicatezza da Pittori è chiamata dolcezza.

Alors, il faut considérer l'homme de deux façons : nu et vêtu. Si nous le dessinons nu, nous pouvons le représenter de deux manières : musclé, ou délicat. Il est à noter, à ce propos, que des peintres appellent cette délicatesse "douceur". ${ }^{42}$

On note que la délicatesse est associée à la douceur, qualité déjà identifiée dans la représentation du putto et liée à l'art de la couleur, et qu'elle s'oppose à une musculature dessinée. Un commentaire de l'Arétin sur une Vénus de Michel Ange permet de préciser les concepts de robustesse et de délicatesse.

L'altro mo è Venere, contornata con maravigliosa rotondità di linee. E perchè tal Dea diffonde le propriétà sue nel desiderio dei due sessi, il prudente uomo le ha fatto nel corpo di femina i muscoli di maschio; talché ella è mossa da sentimenti virili e donneschi con elegante vivacità d'artifizio.

L'autre, maintenant, est une Vénus aux contours merveilleusement arrondis. Comme cette déesse imprègne de ses propriétés le désir de l'un et l'autre sexe, le sage artiste lui a fait des muscles d'homme dans un corps de femme; si bien qu'elle est animée de sentiments à la fois virils et féminins, rendus avec un art qui unit l'élégance à la vivacité. ${ }^{43}$

L'analyse de la Vénus de Michel Ange nous apprend que pour l'Arétin la beauté idéale concilie robustesse et délicatesse, c'est-à-dire les muscles d'un homme dans un corps de femme. Le nu parfait est donc un équilibre entre la vivacité et l'élégance, le dessin et la couleur. Lodovico Dolce va plus loin, et faisant de Michel Ange le maître du nu musclé et de Raphaël ou Titien les maîtres du nu délicat, il affirme sa préférence pour la seconde sorte. Car les nus robustes exigent une bonne connaissance de l'anatomie, mais les nus délicats demandent aussi la maîtrise des lumières et des ombres. C'est en plaçant les "rehauts" et les "reliefs" que l'excellent peintre recouvre les corps de chair et remplit ses nus de grâce :

E se voi qui mi diceste, che ne' ricercamenti de' nudi si conosce, se il Pittore è intendente della Notomia, parte molto bisognevole al Pittore ; perche senza le ossa non si puo formar ne vestir di carni l'huomo: vi rispondo, che'l medesimo si comprende ne gliaccennamenti e macature. E per conchiudere, oltre che all'occhio naturalemente aggradisce piu un nudo gentile e delicato, che un robusto e muscoloso, vi rimetto alle cose de gliantichi : iquali per lo piu hanno usato di far le lor figure delicatissime.

De même, si vous me dites qu'on voit à la façon dont un peintre représente les nus s'il connaît ou non l'anatomie, laquelle est indispensable car l'on ne peut composer, ni vêtir, les chairs de l'homme sans s'appuyer sur

\footnotetext{
${ }^{41}$ Cf. J. N. Grant, "Propertius, Ovid...", p. 48-62.

${ }^{42}$ Lodovico Dolce, Dialogo della pittura..., éd. M.W. Roskill, p. 140 et trad. L. Fallay d'Este, p. 76 (légèrement modifiée).

${ }^{43}$ L'Arétin, Lettres, II, 5, éd. F. Erspamer (Pietro Aretino, Lettere, Parma, Fondazione Pietro Bembo, 1998, vol. II, p. 13-14) et trad. P. Larivaille, Paris, Les Belles Lettres, 2003, p. 47.
} 
l'ossature, je vous réponds qu'on le voit aussi à la façon dont il place les rehauts et les reliefs. Je conclurai en affirmant qu'un nu gracieux et délicat ravit plus l'œil qu'un nu robuste et musclé, et vous renverrai aux maitres de l'antiquité, lesquels ont préféré la première catégorie citée. ${ }^{44}$

La Galatée de Pietro Bembo allie effectivement robustesse et délicatesse. La peinture verbale de la nageuse donne un sentiment de puissance physique : ses bras musclés (lacertis) battent les flots, l'athlétique néréide a vite fait de rejoindre le Faune et le tire sans peine jusqu'à la rive. La jeune nymphe, qui à elle seule a bravé une assemblée de dieux, se paie même le luxe de faire la leçon à Pan qui prétendait la séduire. Toutefois, Galatée plaît aussi par sa délicatesse : mutine, elle incline la tête de côté pour voir les satyres tempêter sur la rive, nageant alors avec une lenteur paresseuse. Les images de sa poitrine ruisselante d'eau et de son pied couleur de neige et auréolé d'écume apportent à sa silhouette des contours gracieux et des notations de couleur qualifiant sa chair. Les mots peignant les parties du corps sont du reste placés systématiquement à la fin du vers, saillant du poème comme s'il jaillissaient de la mer (ore, lacertis, papillae, pede). Erwin Panofsky a montré que les néréides hellénistiques avaient servi souvent de modèle à Titien pour ses nus féminins ${ }^{45}$. Au début des années 1510 , il a peint une Vénus anadyomène (Fig. 3) sur le modèle des Vénus antiques déjà imitées par Giovanni Pisano ou Botticelli. Mais contrairement à ses grandes sœurs toscanes, elle ne cherche plus à cacher sa nudité, tout occupée à essorer sa chevelure. Elle présente des caractéristiques proches de la Galatée de Bembo. Ayant abandonné sa conque, elle gagne en marchant le rivage : ce n'est plus une statue figée, mais une figure en mouvement. Son visage est incliné gracieusement vers l'arrière, la lumière du soleil fait luire sa peau nue et ressortir la blancheur de ses bras robustes et de ses seins. On distingue déjà cependant dans le bas de son corps la tendance du peintre à dissimuler l'ossature et les muscles sous la chair et à arrondir les courbes par le rehaut des ombres. Le Titien est en train d'inventer l'esthétique de la délicatesse qui va faire le succès de ses nus féminins.

\section{III -La maîtresse au lever du lit}

Le second nu féminin des Carmina se trouve dans une élégie personnelle adressée à une femme mariée dont le poète est l'hôte. On est tenté, sans toutefois en avoir de preuve, de songer à Lucrèce Borgia, l'épouse du duc de Ferrare, dont Pietro Bembo s'était épris et à qui est dédiée une autre élégie du même cycle. Le poète néo-latin reprend le thème de la jalousie de l'amant envers l'époux, sur le modèle de l'élégie I, 4 des Amores d'Ovide ${ }^{46}$ :

Cum teneant nos iidem ambo intra tecta penates Vna eadem, atque uno pene etiam in thalamo,

Sape ut nuda sinus et qualis surgit ab ipso Mane toro ueniat conspicienda mihi ;

Non tamen huic possumue meos narrare labores, Atque aliquam aduersis poscere rebus opem;

Non ue sedere una, non dextrae iungere dextram, Non dare lacteolis basiolum digitis :

Omnia qua possunt alii, qui tam nec amantur, Nec quorum tantus concutit ossa Deus.

Quand les mêmes pénates nous tiennent tous deux sous un seul et même toit, et presque aussi dans une seule chambre,

Si bien que souvent, le sein nu, et telle que le matin elle se lève de sa couche, il m'est donné de la voir;

je ne puis pourtant pas lui rapporter mes peines ni demander de l'aide contre l'adversité,

ni m'asseoir avec elle, ni joindre ma main droite à la sienne, ni donner un petit baiser à ses doigts de lait :

\footnotetext{
${ }^{44}$ Lodovico Dolce, Dialogo della pittura..., éd.. M.W. Roskill, p. 142 et trad. L. Fallay d'Este, p. 77.

${ }^{45}$ Cf. E. Panofsky, Le Titien..., p. 164-165 et 225.

${ }^{46} \mathrm{Cf}$. Amores I, 4 et en particulier les vers 1 à 6 : "Vir tuus est epulas nobis aditurus easdem ;/ ultima cena tuo sit, precor, illa uiro./ Ergo ego dilectam tantum conuiua puellam/ aspiciam? tangi quem iuuet, alter erit, alteriusque sinus apte subiecta fouebis? / iniciet collo, cum uolet, ille manum?". Je cite la traduction française de J.P. Néraudau "Ton mari doit assister au même repas que nous : je prie les dieux que ce repas soit le dernier pour ton mari. Ainsi, je devrai me borner à regarder ma bien-aimée, comme un convive quelconque ! Le plaisir de te toucher sera réservé à un autre! Voluptueusement couchée au pied d'un autre, tu réchaufferas son sein. C'est lui qui passera la main autour de ton cou quand il le voudra." (Paris, Les Belles Lettres, 1997, p. 11).
} 
Tout ce que peuvent les autres, qui ne sont pas tant aimés,

Et dont un dieu si grand ne ronge pas les os. ${ }^{47}$

La jeune femme est présentée au lever du lit et dans le désordre de la mise qui suit le sommeil nocturne. Les vers 13 et 14 ont pour source le passage de l'Art d'aimer où Ovide décrit Ariane s'éveillant sur l'île de Dia et découvrant la fuite de Thésée ${ }^{48}$, le texte dont Erwin Panofsky a montré qu'il avait servi de source à Titien pour Bacchus et Ariane, sa dernière Bacchanale ${ }^{49}$. Seulement, Pietro Bembo étend la nudité qu'Ovide cantonnait au pied d'Ariane à tout son sein, siège de la féminité et de la fécondité : l'accusatif de relation sinus est substitué à pedem. Il a pu trouver un autre modèle dans l'élégie I, 3 où Properce aussi avait comparé Cynthia endormie sur son lit à la Gnosienne ${ }^{50}$, si ce n'est que chez le poète antique le réveil de la belle présageait des délices partagés, alors qu'il inflige au poète néo-latin un supplice comparable à celui de Tantale.

\section{-Grâce et vénusté}

Lodovico Dolce se réfère à l'élégie latine et plus particulièrement à Properce pour qualifier les nus peints à son époque :

Percioche molti ve ne fanno alcune, che paiono di Porfido, si nel colore, come in durezza : e le ombre sono troppo fiere, e le piu volte finiscono in puro negro. Molti le fanne troppo bianche, molti troppo rosse. Io per me bramerei un colore anzi bruno, che sconvenevolmente bianco : e sbandirei dalle mie Pitture comunemente quelle guancie vermiglie con le labbra di corallo : perche cosi fatti volti paion mascare. Il bruno si legge essere stato frequentato da Apelle. Onde Propertio riprendendo la sua Cinthia, che adoperava i lisci, dice, che egli disiderava, che ella dimostrasse una tale schietezza e purità di colore, qual si vedeva nelle tavole di Apelle.

De nombreux peintres représentent des carnations que la couleur et la dureté apparente au porphyre, et les ombres sont trop prononcées et le plus souvent elles finissent en noir pur. Beaucoup les font trop blanches, beaucoup d'autres trop rouges. Pour ma part, je préfère le brun à un blanc mal approprié, et je bannirais en général de mes tableaux ces joues rouge vermillon et ces lèvres couleur de corail, qui transforment les visages en masques. On lit qu'Apelle employait fréquemment le brun. Et Properce, réprimandant sa Cynthia qui utilisait des fards, lui dit qu'il désirait que qu'elle montra une netteté et une pureté de teint comparables à celles que l'on voyait dans les tableaux d'Apelle. ${ }^{51}$

L'effet recherché est un éclat naturel qui imite la vie. Pour cette raison, Dolce repousse à la fois les couleurs trop vives qui transforment la figure en masque fardé et les ombres trop prononcées qui lui donnent une dureté sculpturale. Quant à l'idéal de la carnation des figures d'Apelle, il provient en fait de l'élégie 1,2 de Properce où celui-ci, à rebours des préceptes d'Ovide dans l'Art d'aimer ou dans les Cosmétiques, fait l'éloge de la beauté naturelle de la nudité féminine ${ }^{52}$. Pour Properce, la justification de la nudité de Cupidon est purement esthétique : rien ne surpasse l'éclat naturel du teint (candor), tel qu'Apelle savait le reproduire par les nuances du coloris. En hommage à la Vénus du célèbre peintre antique, Lodovico Dolce nomme cet art de la couleur, qu'il croit reconnaître chez Raphaël, "vénusté". Non content d'être passé maître de la délicatesse (delicatezza) et de l'élégance (leggiadria), le peintre aurait fait la reconquête de cette autre qualité, qu'est la "grâce" (charis ou uenustas) :

\footnotetext{
${ }^{47}$ Pietro Bembo, Carmina, XV : "De amica a uiro seruata diligentissime", 11-20 (Turin, 1990, p. 30).

48 Ovide, Ars amatoria, I, 529-530 : "utque erat e somno, tunica uelata recincta, / nuda pedem".

${ }^{49}$ Cf. E. Panofsky, Problems in Titian..., "Titian and Ovid", p. 143 (Le Titien..., "Le Titien et Ovide", p. 203).

${ }^{50} \mathrm{Cf}$. Properce I, 3, 1-10 et en particulier les vers 1-2 et 7-8: "Qualis Tesea iacuit cadente carinal languida desertis Cnosia litoribus [...] talis uisa mihi mollem spirare quietem/ Cynthia non certis nixa caput manibus". Je cite la traduction française de ce passage par S. Viarre : "Telle gisait, tandis que le vaisseau de Thésée s'éloignait, la Gnosienne défaillante sur le rivage solitaire... telle m'apparut Cynthie respirant un doux repos, la tête appuyée sur des mains incertaines" (Paris, Les Belles lettres, 2005, p. 5).

${ }^{51}$ Lodovico Dolce, Dialogo della pittura..., éd. M.W. Roskill, p. 152 et trad. L. Fallay d'Este, p. 82 (traduction modifiée).

52 Properce, I, 2, 8-9 et 21-22 : "Nudus Amor forma non amat artificem./ Aspice quos summittat humus formosa colores [...] sed facies aderat nullis obnoxia gemmis,/ qualis Apelleis est color in tabulis". Je cite la traduction de S. Viarre: "Amour, qui est nu, n'aime pas qui se fait l'artisan de la beauté. Vois les couleurs que fait jaillir une belle terre... mais leur apparence était là sans rien devoir à des pierreries comme le teint sur les tableaux d'Apelle" (Paris, Les Belles Lettres, 2005, p. 4)
} 
e ne acquistò insieme un'altro, che fu chiamato grazioso : percioche oltre la inventione : oltre al disegno : oltre alla varietà : oltre le sue cose tutte movono sommamente : si trova in loro quella parte, che havevano, come scrive Plinio, le figure di Apelle : e questa è la venustà...

Il acquit aussi une autre qualité, qu'on qualifia de "gracieuse". On trouve, en effet, dans ses figures, outre l'invention, le dessin, la diversité et une grande puissance émotive, cette qualité que possédaient, comme l'écrit Pline, les figures d'Apelle. Il s'agit de la vénusté... ${ }^{53}$

À Venise, le nu féminin couché dans un intérieur privé devient manifestement un sujet de peinture apprécié des collectionneurs particuliers ${ }^{54}$. Lodovico Dolce en donne un exemple en décrivant un tableau de Raphaël représentant Roxane nue sur un lit et entourée de l'Amour et d'Hyménée également nus ${ }^{55}$. Vers 1538 , Le Titien a peint une femme nue étendue sur un lit, aujourd'hui conservée à la Galerie des Offices de Florence, et universellement connue sous le nom de Vénus d'Urbin (Fig. 4). Derrière l'alcôve où la belle dévoile son intimité apparaît au second plan une salle somptueuse ou deux femmes de chambres s'affairent pour lui préparer un costume d'apparat, peut-être de noce. Cette figure continue à fasciner par l'éclat incomparable que le peintre est parvenu à rendre, illuminant le ventre et les cuisses, rosissant par du brun le dessin des genoux, des coudes ou la voûte des pieds ${ }^{56}$. Le corps de la Vénus $d^{\prime}$ Urbin a l'air si parfaitement rond et plein qu'il semble vivant et le contraste de couleur avec le blanc terne et froid du drap est quasiment tactile.

Si l'on analyse en détail l'écriture poétique de l'élégie XV de Pietro Bembo, le travail effectué par le poète sur les sonorités de la langue latine peut être comparé à une recherche de couleur vocalique. Dans les vers 12 et 13 par exemple, la modulation des voyelles e, i, u et a crée une harmonie sonore subtile, avec la répétition de la séquence a-i-ou dans nuda sinus et qualis surgit. L'alternance du "a" clair, du "i" brillant et du "ou" plus sombre produit une matière sonore complexe et homogène à la fois qui apparaît comme un équivalent sonore de la chair soyeuse des nus féminins du Titien. Cette couleur tranche par ailleurs avec l'évocation sonore du lit par la voyelle "o" fermée (ab ipso toro). À la fin du distique, l'allitération en "i" (conspicienda mihi) rend l'image de la femme nue encore plus éclatante et mime en quelque sorte son surgissement hors du lit. Plus loin, au vers 18, le teint de la belle est évoqué par la couleur du lait. Le doublet constitué de deux mots de 4 syllabes contenant en leur centre un hiatus (lacteolis basiolum) produit une symétrie particulièrement équilibrée et savoureuse. La projection en fin de vers, une fois de plus, du mot désignant la partie du corps décrite et la répétition du "i" mettent en relief la blancheur de la main, par un effet proche de ces "rehauts" de lumière dont parle Dolce en peinture.

\section{IV -Le martyre}

Le principal exemple de nu masculin dans les Carmina est un nu sacré. L'"Hymnus ad diuum Stephanum" ("Hymne à Saint Stéphane") peint la nudité du premier martyre de la chrétienté. Le poète suit de près les Actes des Apôtres (VII, 54-60) qui rapportent que Stéphane fut jugé et lapidé par la foule pour avoir blasphémé contre Moïse et contre les lois juives, mais que Dieu l'aurait gratifié, à son dernier souffle, d'une théophanie :

Concursu pressum ingenti, saxisque petitum

Nudatos artus, ceu quondam grandine densa

Juppiter ingeminans terram quatit athere ab alto, Crudelis letho dederis nil tale merentem?

Ille quidem placido sustollens lumina uultu Lustrabatque oculis colum, intrepidusque pericli

Laudabat superos, et spe sua damna leuabat: Cui se, quantus erat, manifesta in luce uidendum Ipse pater Diuum dederat cum compare nato Sublimis, medioque illi fulgebat Olympo.

\footnotetext{
${ }^{53}$ Ibidem, éd. M.W. Roskill, p. 174 et trad. L. Fallay d'Este, p. 92.

${ }^{54}$ On accordait à la Renaissance un pouvoir physiologique et quasi magique de fécondité aux icônes du corps dénudé et L.B. Alberti conseillait dans le De Re Adificatoria d'en placer dans la chambre à coucher.

${ }^{55}$ Ibidem, éd. M.W. Roskill, p. 168.

${ }_{56}$ Pour une analyse approfondie du tableau, voir R. Goffen, Titian's Venus of Urbino, New York, Cambridge University Press, 1997 ; O. Calabrese, Venere svelata, La Venere di Urbino di Tiziano, Milan, Silvana, 2003 et encore A. Colantuono, Titian, colonna..., "A Venus in the Bedroom", p. 245-257.
} 
Poussé par une foule immense et criblé de pierres,

son corps nu, comme parfois d'une grêle serrée

Jupiter frappe la terre à doubles coups depuis les hauteurs de l'éther,

tu lui as donné une mort cruelle, à lui qui ne méritait rien de tel ?

Lui pourtant, levant les yeux, le visage paisible,

Parcourait le ciel du regard, et sans trembler devant le danger

Il louait les cieux et allégeait ses maux par l'espoir.

À lui, dans toute sa grandeur, s'était donné à voir en pleine lumière

Dieu le Père en personne, avec son fils pareil à lui,

Sublime, et il resplendissait pour lui au milieu de l'Olympe..$^{57}$

\section{-Terribilità et agrément}

Cette description du martyre de Saint Stéphane illustre à la perfection l'idéal de "terribilità" tempéré par l'agrément que propose Lodovico Dolce : dolci.

Rafaello ne ha fatto di ogni sorte, e di piacevoli, e di terribili e ricercati, benche con atti piu temperati e piu

Raphaël a fait des nus de toutes sortes, agréables et recherchés, pleins de terribilità, bien que plus tempérés et plus doux par leurs gestes. ${ }^{58}$

On sait grâce aux travaux de David Summers que le concept de terribilità provient de la rhétorique grecque ${ }^{59}$. Développée notamment par les rhéteurs Hermogène, Démétrius et Denys d'Halicarnasse, la deinotès unit les idées de grandeur, de force d'expression et d'artifice. Au Moyen Âge, elle fut aussi associée à la difficulté et à la stupeur et elle a toujours été considérée comme le style approprié aux sujets sacrés. Ici, toute la première partie de l'hymne vise à produire l'effroi. Pietro Bembo met en œuvre l'arsenal rhétorique antique de la "grauitas" et de la "uehementia". Mais l'on croit reconnaître aussi dans ces vers le "style tragique" qui consiste, selon Hermogène de Tarse a donner de la grandeur à un sujet médiocre par l'expansion et l'abondance de l'expression ${ }^{60}$. La syntaxe est véhémente et fait preuve de "recherche" -Hermogène parlerait de "complication"- à cause des appositions et de la comparaison qui diffèrent l'action principale. Le vocabulaire, solennel et terrible, est celui de la "grauitas": l'expression "crudelis letho" donne une couleur sombre à la scène. La comparaison de la lapidation du corps nu de Stéphane avec un orage de grêle précipité du ciel sur la terre par Jupiter tonnant achève de susciter la terreur. Dans les vers 10 à 12 l'allitération en dentales ("nudatos artus", "quondam grandine densa" ou "terram quatit aethere ab alto", et parfait à redoublement "dederit") et en occlusives ("ceu", "quondam", "quatit", "crudelis") figurent par les sonorités les coups qui s'abattent sur la chair du corps supplicié. La seconde partie du texte, au contraire, dépeint la sérénité du saint caractérisé par les adjectifs "placido" et "intrepidans". S'identifiant au personnage par un mouvement de sympathie, le lecteur goûte l'apaisement des souffrances de la mort grâce à l'espérance et à la foi chrétienne. Cet allègement de la peine est figuré par la théophanie : le tableau s'éclaire avec l'apparition du Père et du fils en gloire ("lumina", "manifesta in luce", "fulgebat"). Cette image sublime et rayonnante du Dieu chrétien contraste fortement avec celle, sombre et terrible, de Jupiter. Parallèlement, l'éclaircissement des voyelles mime l'apaisement de l'âme, le "a" clair l'emportant sur le "e" fermé (spe sua damna leuabat). Une série de parallélismes à l'initiale du vers ou d'échos internes produisent un effet plaisant de symétrie ("lustrabat/ laudabat" ; "cui se/ ipse" ; "erat /dederat"). Dans l'"Hymne à Saint Stéphane" de Pietro Bembo, l'évocation de la nudité du martyre a pour effet de susciter les deux affects de terreur et de pitié.

Thomas Puttfarken a noté que l'évolution du style de Titien de l'agrément vers la "terribilità" a pris un tournant décisif dans les années 1540, époque où ses liens se sont resserrés avec Pietro Bembo ${ }^{61}$. De fait, les commentateurs ont souligné l'agrément des premiers nus sacrés du Titien. L'Arétin a loué avec enthousiasme la douceur, la délicatesse et l'éclat du teint d'un Christ à la colonne :

\footnotetext{
${ }^{57}$ Pietro Bembo, Carmina, XX : "Hymnus ad diuum Stephanum", 9-18 (Turin, 1990, p. 49).

${ }^{58}$ Lodovico Dolce, Dialogo della pittura..., éd. M.W. Roskill, p. 172 et trad. L. Fallay d'Este, p. 91.

${ }^{59}$ D. Summers, Michelangelo and the Language of Art, Priceton, Princeton University Press, 1981, p. $234-241$.

${ }^{60}$ Hermogène de Tarse, Sur les idées, p. 450 (trad. M. Patillon, p. 544-545)

61 T. Puttfarken, Titian \& Tragic painting..., p. 70
} 
Egli era più che di carne e d'ossa. E con la purità del suo corpo, vinceva il candido de i gigli colti negli orti superni. La sua statura era mezzana. Aveva le braccia spedite, le spalle larghe, e il petto si univa al corpo dolcissimamente. Aveva le coscie tonde, le gambe svelte, e i piu bei piedi e le più belle mane che si vedesser mai. E tutto il suo corpo insieme pareva una composizione d'avorio che respirasse, sul quale la natura avesse spruzzato il rosato delle gote de l'Aurora.

Il était plus qu'un homme en chair et en os, et la pureté de son corps l'emportait sur la candeur des lys cueillis dans les jardins célestes. Il était de taille moyenne. Il avait les bras élancés, les épaules larges, et sa poitrine s'unissait au corps avec une extrême douceur. Il avait les cuisses rondes, les jambes sveltes et les plus beaux pieds, les plus belles mains que l'on eût jamais vus. Son corps tout entier ressemblait à une statue d'ivoire qui eût respiré et sur laquelle la nature eût vaporisé la teinte rosée des joues de l'Aurore... ${ }^{62}$

De même, Lodovico Dolce rend hommage au coloris de la chair d'un Saint-Sébastien, peint par Titien dans l'Église Saint Nicolas et actuellement conservé au Vatican :

Un San Sebastiano ignudo di bellissima forma, e con una tinta di carne cosi simile alla evvera, che non par dipinto, ma vivo. Ilqual San Sebastiano essendo il Pordenone andato a vedere, hebbe a dire, io stimo, che Titiano in quel nudo habbia posto carne, e non colori.

Un Saint Sébastien nu, dont la forme est magnifique et la carnation tout à fait naturelle ; on dirait qu'il n'est pas peint, mais bien vivant. Porderone déclara, après avoir vu ce Saint Sébastien : "J'estime que Titien a mis de la chair et non des couleurs dans ce nu".63

Cette carnation naturelle qu'il admire dans la figure de Saint Sébastien n'est pas très éloignée de la vénusté dont on vient de voir qu'elle caractérisait les nus féminins de Titien. En revanche, le dernier Saint Sébastien qu'il peignit à la fin de sa vie (Fig. 5, c. 1570-75), beaucoup plus pathétique, présente un certain nombre de similitudes avec l'"Hymnus ad diuum Stephanum" de Bembo. L'attitude de la figure est comparable, le visage levé et scrutant les cieux du regard. Le fond sur lequel elle se distingue est un ciel d'orage tourmenté et terrifiant, comme dans la description du poète. Enfin et surtout, le peintre a renoncé au clairobscur pour produire des contrastes violents de lumière et d'ombre ${ }^{64}$. La clarté de la poitrine nue exposée aux coups tranche avec l'ombre dans laquelle disparaissent les bras liés dans le dos et la jambe qui se replie sous l'effet de la douleur et de la peur. Les nuages noirs et la terre sombre l'emportent sur la lumière rougeoyante du crépuscule. L'esthétique tragique de la "terribilità" ne laisse désormais que peu de place à l'agrément du spectateur. Le Martyre de Saint Laurent, peint en 1557 à Venise pour l'église Santa Maria, puis dans une seconde version pour Philippe II en 1564, va plus loin encore dans l'expression de la cruauté. Comme dans l'"Hymne à Saint Stéphane" le martyre est seul, perdu et aveuglé, face à la fureur d'une foule hostile. Cette situation suscite à elle seule la pitié du spectateur. Le Titien a aussi exploité les virtualités picturales du supplice de Saint Laurent, brûlé sur des braises. Dans cette scène nocturne, la lumière changeante du feu accuse le relief du corps, dessinant les muscles tordus par la douleur. L'éclat blafard de la nudité n'a plus rien désormais de la douceur, de la délicatesse ou de la grâce qui faisaient jadis la célébrité du peintre.

Je conclurai avec un dernier exemple de nu tiré de la prière du "Faunus ad Nympeum fluuium" ("Faune au fleuve Nymphée"). Accusant le fleuve de souiller la pureté de son sanctuaire, le dieu Pan peint une fresque des débauches qui se déroulent sur ses rives, à l'imitation des bains de l'antique Baiès :

Huc ueniunt mixtce pueris de more puella,

Inque tua, posita ueste, natatur aqua.

Lusibus interea duxisse procacibus horas

Nequitiaque iuuat continuare diem :

80

Cumque libet, mediis passim iunguntur in undis,

${ }^{62}$ L'Arétin, Umanità di Cristo, dans Pietro Aretino, introd. G. Ferroni, Rome, Istituto poligrafico e Zecca dello Stato, 2002, p. 387 et trad. P. Larivaille, p. 6.

${ }^{63}$ Lodovico Dolce, Dialogo della pittura ..., éd. M.W. Roskill, p. 188-189 et trad. L. Fallay d'Este, p. 99.

${ }^{64}$ D. Summers a montré que des procédés esthétiques comme le contrapposto ou la figura serpentinata, caractéristique de certains nus masculins de la Renaissance, en particulier chez Michel-Ange et le Titien, étaient dérivés de la figure rhétorique de l'antithesis, transmise à la peinture et à la sculpture notamment à travers les notions de uarietas chez Alberti et de ricerca chez Lodovico Dolce (D. Summers, "Contrapposto : Style and Meaning in Renaissance Art", The Art Bulletin, 59 (1977), p. 336-361. 
Lasciuum femori conseriturque femur.

Tu tamen hac pateris, nec te mea numina tangunt, Ante oculos fieri turpia probra meos.

Quin etiam si quid specto, et spectare necesse est, Illi se improbius liberiusque petunt.

Permolitur pueroque puer, pactique uicissim Obscanos agitant nostra sub ora modos

Meque uocans uda media inter sauia lingua, Crissat ab imposito fixa puella mare.

At mihi tum mediae saliunt tentigine uena, Surgit et in cornu spina recurua suum.

Ici, les jeunes filles ont coutume de venir mêlées aux jeunes gens, Et l'on nage dans ton eau, dépouillé de vêtements.

On aime avoir passé des heures à des jeux impudents Et poursuivre la journée avec indolence.

Quand ils veulent, ils se rejoignent, ici où là, au milieu des ondes Et la cuisse lascive s'unit à une autre cuisse.

Toi pourtant tu permets cela, et ma puissance divine ne t'émeut pas,

Ni que ces scandaleuses débauches aient lieu sous mes yeux.

Bien pire, si je regarde, et l'on regarde nécessairement, Ils se cherchent avec plus d'audace et de licence

Un jeune homme est besogné par un jeune homme et, s'engageant mutuellement, ils battent sous nos yeux des cadences obscènes.

M'appelant d'une langue humide au milieu des délices, Une jeune fille se tortille clouée sous un mâle.

Mais alors les veines me battent au milieu du corps sous la tension Et dans sa corne surgit l'épine recourbée. ${ }^{65}$

La nudité est d'abord décrite négativement, grâce à la participiale "posita ueste" (v. 8), puis positivement avec la double occurrence du mot "femur", placé à la césure et à la fin du pentamètre (v. 12). Ce tableau d'une licence et d'une précision rares est autorisé du fait que le personnage-narrateur l'a condamné d'emblée comme une infamie. Pourtant, le Faune, dont la profession comme garant de la pudeur ne peut être prise longtemps au sérieux, reconnaît peu à peu sa participation à la scène. Il se donne d'abord le statut de spectateur ("ante oculos meos", "specto"), fût-ce un spectateur forcé ("spectare necesse est"). Mais bientôt il doit constater les effets du voyeurisme sur les protagonistes, dont il ne fait qu'encourager l'audace, ainsi que sur son propre corps, éveillé au désir.

On songe bien sûr aux scènes lascives des Andriens ou de la Vénus du Pardo du Titien, mais par son fonctionnement et par la réflexion qu'elle engage sur la contemplation du nu, cette élégie peut être aussi rapprochée d'autres scènes de bains représentées par le même peintre, comme Diane et Actéon (Fig. 6) et Diane et Callisto. On sait que les fresques de Michel Ange dans la Chapelle Sixtine ont suscité à Venise un débat sur le decorum à propos de la représentation du nu. L'Arétin, scandalisé par la liberté des inventions de Michel Ange, condamne sans appel le dévoilement des organes génitaux des saints, accusant le peintre de prendre la chapelle du Vatican pour un établissement de bain. Et Lodovico Dolce d'invoquer précisément l'autorité du cardinal Bembo comme gardien des bonnes mœurs et du caractère sacro-saint de la basilique Saint Pierre :

Chi ardirà di affermar, che stia bene, che nella Chiesa di San Pietro, Prencipe de gli Apostoli, in una Roma, ove concorre tutto il mondo : nella cappella del Pontefice ; ilquale, come ben dice il Bembo, in terra ne assembra Dio, si veggano dipinti tanti ignudi, che dimostrano dishonestamente dritti e riversi.

Qui osera affirmer qu'il est bien de voir dans l'église de Saint Pierre, prince des Apôtres, dans une Rome où accourt le monde entier, dans la chapelle du pontife, lequel, comme Bembo le dit si bien, représente Dieu sur terre, tous ces nus qui montrent avec indécence leur derrière et leur devant. ${ }^{66}$

Quand on vient de lire les vers des Carmina, on ne peut s'empêcher de sourire, pourtant il se pourrait que Titien ait retenu, lui, la leçon du Faune-Bembo dans ses deux poesie sur le mythe de Diane peintes entre 1556 et 1559 pour le roi d'Espagne. Dans Diane et Actéon (Fig. 6), le jeune chasseur est amené malgré lui à contempler la nudité des baigneuses et sa participation

\footnotetext{
${ }^{65}$ Pietro Bembo, Carmina, II : "Faunus ad Nympeum fluuium, fictum pro antiquo", 77-92 (Turin, 1990, p. 9).

${ }^{66}$ Lodovico Dolce, Dialogo della pittura..., éd. M.W. Roskill, p. 162 et trad. L. Fallay d'Este, p. 86.
} 
à la scène par le seul regard suffit à le rendre coupable. Le personnage de dos au premier plan du tableau apparaît comme une projection du spectateur. Mieux, dans Diane et Callisto, la déesse de la Chasteté en personne devient voyeuse: elle fait dénuder la nymphe pour constater le crime de ses yeux. Le poète comme le peintre révèlent la périlleuse limite fixée par le "decorum" entre espace sacré et espace profane et ils symbolisent celle-ci par un cours d'eau. Tous deux font prendre conscience au lecteur-spectateur qui se délecte de la représentation d'un nu de la réversibilité des points de vue et de la facilité avec laquelle le regard transgresse cette frontière. Réinventant le nu antique, l'écrivain et l'artiste ont fait par eux-mêmes l'épreuve de son pouvoir subversif et se sont heurtés à l'interdit sacré ancestral. 
Table des illustrations

Fig. 1-Titien, Danaé, Naples, Galleria Nazionale di Capodimonte.

Fig. 2- Titien, L'Offrande à Vénus, Madrid, Museo del Prado.

Fig. 3- Titien, Vénus anadyomène, Édimbourg, National Gallery of Scotland.

Fig. 4- Titien, La Vénus d'Urbino, Florence, Musée des Offices.

Fig. 5- Titien, Saint Sébastien, Saint Pétersbourg, Musée de l'Ermitage.

Fig. 6- Titien, Diane et Actéon, Édimbourg, National Gallery of Scotland. 